\title{
Nonsingular Einsteinian Cosmology: How Galactic Momentum Prevents Cosmic Singularities
}

\author{
Kenneth J. Epstein \\ 6400 N. Sheridan No. 2604, Chicago, IL 60626-5331, USA \\ Correspondence should be addressed to Kenneth J. Epstein; kennethepstein@sbcglobal.net
}

Received 5 June 2013; Accepted 27 June 2013

Academic Editor: Sergei Odintsov

Copyright (C) 2013 Kenneth J. Epstein. This is an open access article distributed under the Creative Commons Attribution License, which permits unrestricted use, distribution, and reproduction in any medium, provided the original work is properly cited.

It is shown how Einstein's equation can account for the evolution of the universe without an initial singularity and can explain the inflation epoch as a momentum dominated era in which energy from matter and radiation drove extremely accelerated expansion of space. It is shown how an object with momentum loses energy to the expanding universe and how this energy can contribute to accelerated spatial expansion more effectively than vacuum energy, because virtual particles, the source of vacuum energy, can have negative energy, which can cancel any positive energy from the vacuum. Radiation and matter with momentum have positive but decreasing energy in the expanding universe, and the energy lost by them can contribute to accelerated spatial expansion between galactic clusters, making dark energy a classical effect that can be explained by general relativity without quantum mechanics, and, as (13) and (15) show, without an initial singularity or a big bang. This role of momentum, which was overlooked in the Standard Cosmological Model, is the basis of a simpler model which agrees with what is correct in the old model and corrects what is wrong with it.

\section{Introduction}

The Standard Cosmological Model entails a space-time metric with line element [1-4]

$$
d s^{2}=c^{2} d t^{2}-a^{2}(t)\left[d r^{2}+r^{2} d \theta^{2}+r^{2} \sin ^{2}(\theta) d \varphi^{2}\right],
$$

where $c$ is the vacuum speed of light relative to a local Lorentz frame, $t$ is cosmic time, $a(t)$ is the time-dependent scale factor of the Universe, and $r, \theta, \varphi$ are spherical coordinates of a spatially flat (Euclidean) 3-space. A particle of proper mass $m$ has the Hamiltonian $[5,6]$

$$
\begin{aligned}
& H\left(r, \theta, t, p_{r}, p_{\theta}, p_{\varphi}\right) \\
& \quad=c \sqrt{m^{2} c^{2}+\frac{p_{r}^{2}}{a^{2}}+\frac{p_{\theta}^{2}}{r^{2} a^{2}}+\frac{p_{\varphi}^{2}}{r^{2} \sin ^{2}(\theta) a^{2}}},
\end{aligned}
$$

where $p_{r}, p_{\theta}$, and $p_{\varphi}$ are canonical momenta conjugate to canonical coordinates $r, \theta$, and $\varphi$, respectively. The azimuthal angular momentum $p_{\varphi}$ is conserved because $H$ does not depend on $\varphi$, but $H$ is not conserved unless all three momenta vanish. If $p_{\varphi}=0$, the polar angular momentum $p_{\theta}$ is conserved because $H$ no longer depends on $\theta$. If $p_{\theta}=p_{\varphi}=0$, the radial momentum $p_{r}$ is conserved because $H$ no longer depends on $r$. Defining $p_{r} \equiv p, H$ then reduces to

$$
H(p, t)=c \sqrt{m^{2} c^{2}+\frac{p^{2}}{a^{2}(t)}},
$$

showing how a particle with momentum loses energy to the expanding Universe [5].

A logical question, then, is where does that energy go? A logical answer is that it goes to the Hamiltonian of the Universe, which, in a one-dimensional minisuperspace model, can be expressed in geometrized units as the conserved quantity [7]

$$
\mathscr{H}=\mathscr{H}(P, x)=\frac{P^{2}}{2 M}+M \mathscr{U}(x),
$$


with canonical momentum $P$, canonical coordinate $x$, effective mass $M$, and potential

$$
\begin{aligned}
\mathcal{U}(x)= & \left(\frac{9 k}{8}\right)\left(\frac{x}{\alpha}\right)^{2 / 3}-\frac{3 \Lambda x^{2}}{8} \\
& -3 \pi \rho_{m o} x_{o}^{2}-3 \pi \rho_{r o} x_{o}^{2}\left(\frac{x_{o}}{x}\right)^{2 / 3},
\end{aligned}
$$

where $k$ is the spatial curvature constant $( \pm 1$ or 0$), \alpha$ is a constant such that $a=\alpha^{1 / 3} x^{2 / 3}, \Lambda$ is Einstein's cosmological constant, $\rho_{m o}$ and $\rho_{r o}$ are energy densities of matter and radiation, respectively, at some initial time $t_{o}$, and $x_{o}=x\left(t_{o}\right)$. The first term of $\mathcal{U}$ is eliminated by metric (1), for which $k=0$. The second term gives exponentially accelerated expansion of a vacuum dominated Universe, with $\Lambda$ attributed to dark energy due to vacuum fluctuations, a quantum effect. The third term gives $x \propto t$, and $a \propto t^{2 / 3}$, the Einstein-de Sitter scale factor of a matter dominated Universe. The fourth term gives $a \propto t^{1 / 2}$ in a radiation dominated Universe. The constraint $\mathscr{H}=0$ gives the same differential equation for the expansion factor $a(t)$ as is obtained from the Standard Cosmological Model $[4,7]$.

However, $\mathscr{H}$ is defective, because the Standard Cosmological Model is defective. $\rho_{\text {mo }}$ only includes the rest mass $m$ of an object whose relativistic mass is Hamiltonian (3) with momentum $p$. This deficiency is corrected here by revising $\mathscr{H}$ to the form

$$
K(P, x, p)=\frac{P^{2}}{2 M}+\sum_{n} \sqrt{m_{n}^{2}+\frac{p_{n}^{2}}{a^{2}}}-\frac{3 M \Lambda x^{2}}{8},
$$

where $a^{2}=\alpha^{2 / 3} x^{4 / 3}$ in terms of the cosmic coordinate $x$, thereby conserving $K$ by making $\partial K / \partial t=0$. The sum over $n$ is taken over all objects $m_{n}$, whose momenta $p_{n}$ create an interaction with the Universe [7], so they are no longer treated like test particles. $p$ denotes all the $p_{n}$, which remain constant because $K$ is independent of the radial coordinates $r_{n}$.

\section{Canonical Equations}

Hamiltonian (6) is an example of scientific induction [8], from which rigorous mathematical deduction, in the form of Hamilton's equations, gives the coordinate velocities

$$
\frac{d r_{n}}{d t}=\frac{\partial K}{\partial p_{n}}=\frac{a^{-1} p_{n}}{\sqrt{a^{2} m_{n}^{2}+p_{n}^{2}}}
$$

$d x / d t=\partial K / \partial P=P / M$. Defining $\ell_{n} \equiv a r_{n}$ as the proper or physical distance of object $n$ from a real or hypothetical observer and dropping the subscript $n$, give the recession velocity

$$
\frac{d \ell}{d t}=r \frac{d a}{d t}+\frac{p}{\sqrt{a^{2} m^{2}+p^{2}}},
$$

the Hubble velocity increased by positive $p$ and decreased by negative $p$.
Using an overdot to denote $d / d t$ and substituting $P=M \dot{x}$ in (6), give

$$
\frac{1}{2} M \dot{x}^{2}=K+\frac{3}{8} M \Lambda x^{2}-\sum_{n} \sqrt{m_{n}^{2}+\alpha^{-2 / 3} p_{n}^{2} x^{-4 / 3}},
$$

indicating that $\dot{x}$ is a monotonically increasing function of $x$, and $\ddot{x}$ is positive definite. But this mathematical acceleration does not necessarily imply physical acceleration. Expressing (9) in terms of the scale factor $a$ through the relation $x=$ $\alpha^{-1 / 2} a^{3 / 2}$ gives

$$
\frac{9}{8} M \dot{a}^{2}=K \alpha a^{-1}+\frac{3}{8} M \Lambda a^{2}-\alpha a^{-1} \sum_{n} \sqrt{m_{n}^{2}+p_{n}^{2} a^{-2}},
$$

where the first term on the right $\left(K \alpha a^{-1}\right)$ is a monotonically decreasing function of $a$, while the second and third terms are monotonically increasing, so $\ddot{a}$ can be positive or negative. Mathematics is simpler in terms of the canonical coordinate $x$ [7], but physics is clearer in terms of the expansion factor $a$. The time derivative of (10) gives the acceleration

$$
\ddot{a}=\frac{\Lambda a}{3}+\frac{4 \alpha}{9 M a^{2}} \sum_{n} \frac{m_{n}^{2}+2 p_{n}^{2} a^{-2}}{\sqrt{m_{n}^{2}+p_{n}^{2} a^{-2}}}-\frac{4 K \alpha}{9 M a^{2}} .
$$

Defining the conserved quantities

$$
\mathscr{M} \equiv \sum_{n} m_{n}, \quad \mathscr{P} \equiv \sum_{n}\left|p_{n}\right|
$$

conserved because the $m_{n}$ and $p_{n}$ are constant; Hamiltonian (6) allows a momentum dominated epoch at very early times, a matter dominated epoch at intermediate times, and a vacuum dominated epoch at later times. Acceleration (11) is positive in the momentum era, negative in the matter era, and positive again in the vacuum era.

\section{Momentum Dominated Epoch}

Momentum domination occurs when $\Lambda$ and the $m_{n}$ can be neglected in (10) and (11), giving

$$
\begin{aligned}
& \frac{9}{8} M \dot{a}^{2}=K \alpha a^{-1}-\mathscr{P} \alpha a^{-2}, \\
& \frac{9}{4} M \ddot{a}=2 \mathscr{P} \alpha a^{-3}-K \alpha a^{-2} .
\end{aligned}
$$

$\ddot{a}$ vanishes at scale factor $a_{1}=2 \mathscr{P} / K$, the first inflection point, with $\ddot{a}>0$ for $a<a_{1}$ and deceleration for $a>a_{1}$. $\dot{a}$ vanishes at scale factor $a_{o}=\mathscr{P} / K$ and is positive definite for $a>a_{o}$ but imaginary for $a<a_{o}$, which is thereby forbidden by (13) as physically impossible. Thus, the momentum factor $\mathscr{P}$ rules out a singularity by requiring the Universe to be created spatially flat with initial scale factor $a_{o}>0$-the ultimate example of inflation-after which no further inflation is needed to achieve flatness or size. But (14) indicates that accelerated expansion continues for $a_{0}<a<a_{1}$, after which deceleration sets in. 
Equation (13) can be integrated to give

$$
\left(a+2 a_{o}\right) \sqrt{a-a_{o}}=2 \alpha K M^{-1} t,
$$

with the initial time being defined so that $a=a_{o}$ when $t=0$. Equation (15) confirms the impossibility of the scale factor $a(t)$ being less than $a_{o}$, since that would make $t$ imaginary.

The momentum factor $\mathscr{P}$, defined in (12), is essential for these results and is independent of the signs of the $p_{n}$ in (6). This momentum symmetry, together with the time reversal symmetry of Einstein's equation, allows a contracting Universe to undergo a smooth bounce $[\dot{a}(0)=0]$ at the minimum scale factor $a_{o}$ and then rebound from it as if it had been created at $t=0$.

Unlike Hamiltonian $\mathscr{H}$ of (4), Hamiltonian $K$ of (6) need not vanish. $K>0$ is necessary for accelerating expansion $(\ddot{a}>0)$ in the momentum dominated era $\left(a_{o}<a<a_{1}\right)$.

The recession velocity $(8)$ reduces to the form

$$
\dot{\ell}=\dot{a} r+p|p|^{-1}
$$

indicating that $|p|$ is assumed to be so large that the motion of the receding object approximates that of a massless particle moving at luminal speed during this era. In this respect, it is like a radiation era but with repulsive radiation, rather than the attractive radiation of Hamiltonian (4). But the end result is the same, as the Universe expands and matter domination sets in.

\section{Matter and Vacuum Dominated Epochs}

When the $p_{n}$ can be neglected for $t \geq \tau$ (the starting time of matter domination), (10) gives

$$
\frac{9}{8} M \dot{a}^{2}=(K-\mathscr{M}) \alpha a^{-1}+\frac{3}{8} M \Lambda a^{2}
$$

For $t \geq \tau$, (17) is then readily integrated to give

$$
a(t)=2\left[\frac{(K-\mathscr{M}) \alpha}{3 M \Lambda}\right]^{1 / 3} \sinh ^{2 / 3}\left(\frac{\sqrt{3 \Lambda}}{2} t\right)
$$

Defining $T$ as the transition time from matter domination to vacuum domination, it follows that, for $\tau \leq t \leq T$ and $(\sqrt{3 \Lambda} / 2) T \ll 1,(18)$ gives the Einstein-de Sitter scale factor

$$
a(t)=\left[\frac{2(K-\mathscr{M}) \alpha}{M}\right]^{1 / 3} t^{2 / 3}
$$

with $\dot{a}>0$ and $\ddot{a}<0$ in the matter dominated era. For $(\sqrt{3 \Lambda} / 2) t \gg 1$, (18) gives

$$
a(t)=\left[\frac{(K-\mathscr{M}) \alpha}{3 M \Lambda}\right]^{1 / 3} \exp \left(\frac{\sqrt{\Lambda} t}{\sqrt{3}}\right)
$$

the de Sitter scale factor, with $\dot{a}>0$ and $\ddot{a}>0$ in the vacuum dominated era, so called because $\Lambda$, which occurs naturally in Einstein's equation, is a classical property of the vacuum, whose quantum fluctuations are not invoked here because virtual particle-antiparticle pairs created spontaneously from the vacuum can have positive or negative energy [9], making it uncertain whether such vacuum fluctuations can explain $\Lambda$, since their contributions to positive and negative $\Lambda$ may be canceled.

\section{Conclusions}

The initial singularity of the Standard Model comes from neglecting the conserved momenta $p_{n}$ in the relativistic mass terms of Hamiltonian (6). When the $p_{n}$ are included, Einstein's equation forbids a singularity, thereby disproving the singularity theorems $[1,3,10]$. This quantum leap in cosmology is achieved within the framework of general relativity, through the classical mechanism of momentum, without quantization or any non-Einsteinian effects. It does not improve on Einstein's theory, but proves that Einstein's theory is much better than it was thought to be. Other models based on a nonsingular bounce followed by expansion are not strictly Einsteinian, because they invoke other mechanisms [11] in lieu of the $p_{n}$, whereas this galactic momentum is the essential mechanism of nonsingular Einsteinian cosmology.

\section{References}

[1] G. F. R. Ellis, R. Maartens, and M. A. H. MacCallum, Relativistic Cosmology, Cambridge University Press, Cambridge, UK, 2012.

[2] S. Weinberg, Cosmology, Oxford University Press, Oxford, UK, 2008.

[3] R. M. Wald, General Relativity, University of Chicago Press, Chicago, Ill, USA, 1984.

[4] C. W. Misner, K. S. Thorne, and J. A. Wheeler, Gravitation, Freeman, New York, NY, USA, 1973.

[5] K. J. Epstein, "Hamiltonian geometrodynamics," Physics Essays, vol. 5, no. 1, pp. 133-141, 1992.

[6] K. J. Epstein, "Hamiltonian approach to frame dragging," General Relativity and Gravitation, vol. 40, no. 7, pp. 1367-1378, 2008.

[7] K. J. Epstein, “The quantum connection,” Physics Essays, vol. 15, no. 4, pp. 418-421, 2002.

[8] D. Harriman, The Logical Leap: Induction in Physics, New American Library, New York, NY, USA, 2010.

[9] L. Boi, The Quantum Vacuum, Johns Hopkins University Press, Baltimore, Md, USA, 2011.

[10] S. W. Hawking and G. F. R. Ellis, The Large Scale Structure of Space-Time, Cambridge University Press, Cambridge, UK, 1973.

[11] S. E. Perez Bergliaffa, "Nonsingular cosmological models," http:// arxiv.org/abs/1105.5424. 

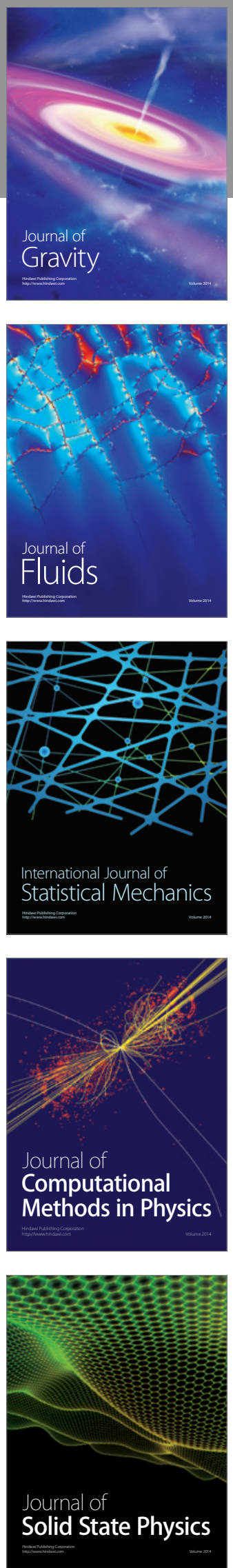

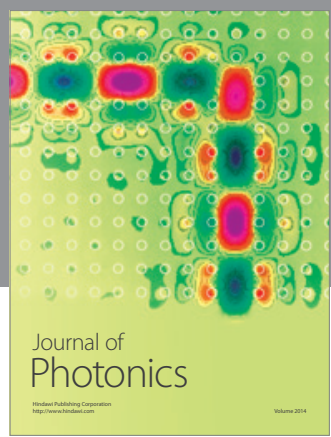

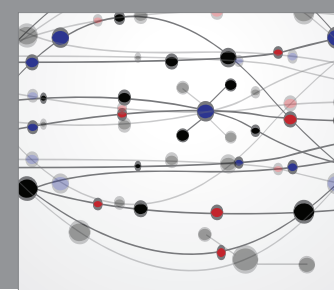

The Scientific World Journal

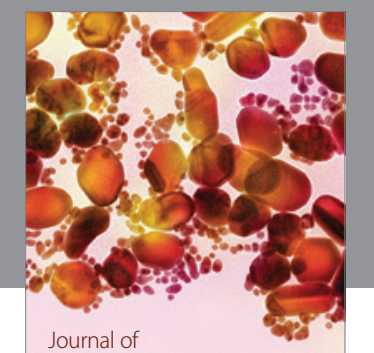

Soft Matter
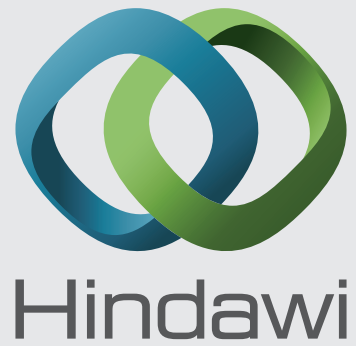

Submit your manuscripts at

http://www.hindawi.com
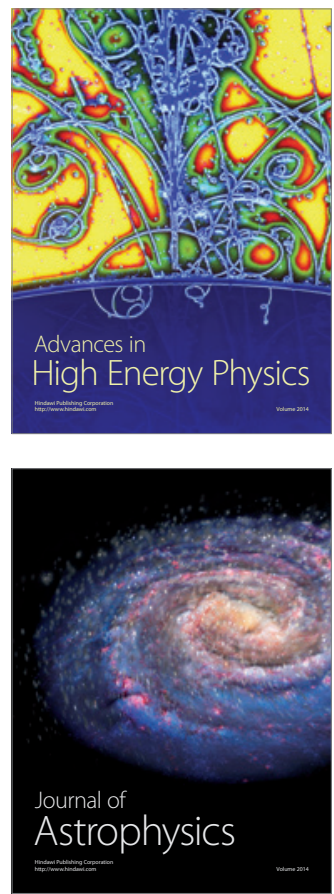
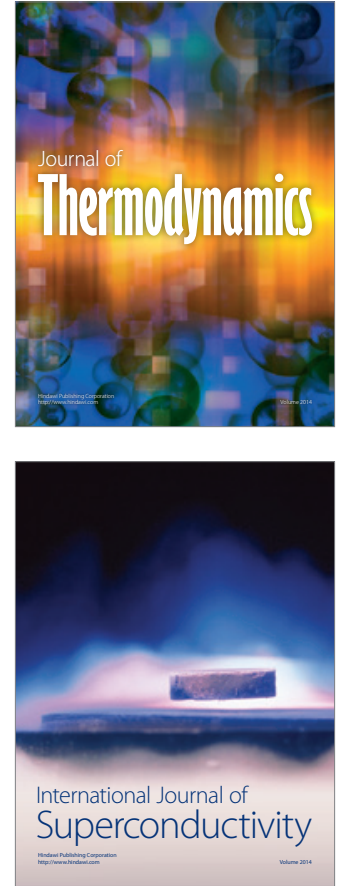
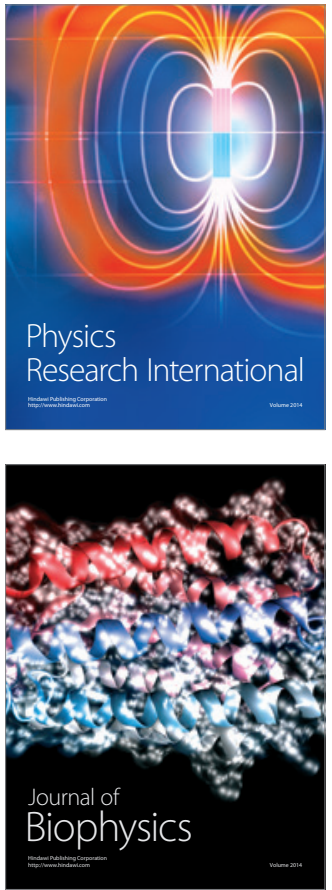
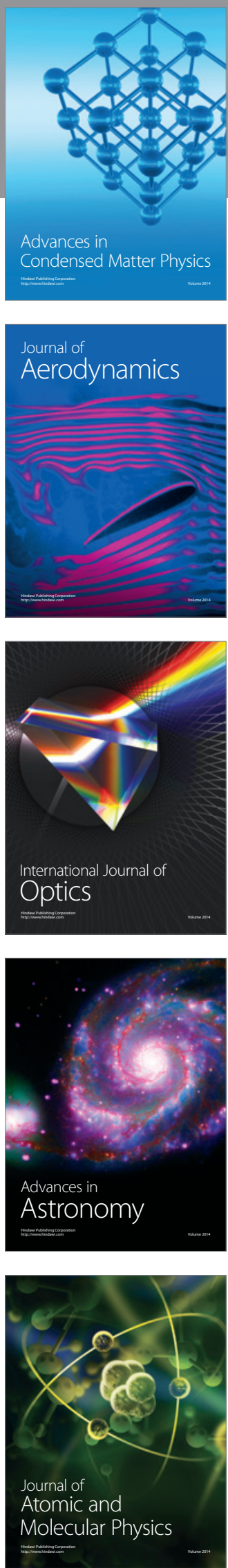